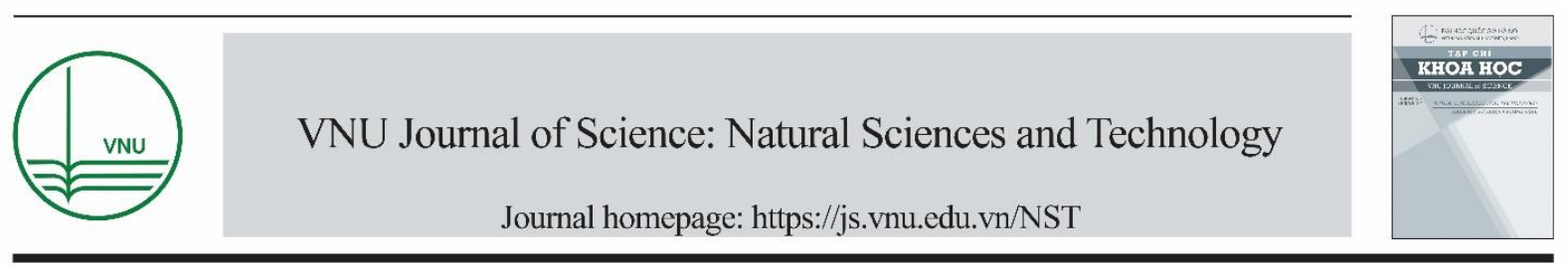

Original Article

\title{
The Morphological Measurement Variations of Periophthalmus chrysospilos along the Coastline in the Mekong Delta
}

\author{
Le Trung Hieu ${ }^{1}$, Dinh Minh Quang, ${ }^{2, *}$, Hua Van $\mathrm{U}^{3}$, Nguyen Huu Duc Ton ${ }^{2}$ \\ ${ }^{1}$ Tran Dai Nghia High School, Hung Phu, Cai Rang, Can Tho City, Vietnam \\ ${ }^{2}$ Can Tho University, 3/2 Street, Xuan Khanh, Ninh Kieu, Can Tho City, Vietnam \\ ${ }^{3}$ Nguyen Thi Minh Khai High School for the Gifted, No. 1 Ho Nuoc Ngot, \\ No. 6 Ward, Soc Trang City, Soc Trang, Vietnam
}

Received 30 May 2021

Revised 24 July 2021; Accepted 15 August 2021

\begin{abstract}
This study provided data on morphological variations of Periophthalmus chrysospilosin four provinces from Tra Vinh to Soc Trang, Bac Lieu, and Ca Mau. Fish samples were collected by hand-catching over 12 months from April 2020 to March 2021. Analyzed results of a collection of 1,031 individuals (508 females and 523 males) showed that the total length (TL) and weight $(W)$ of the female Periophthalmus chrysospilos were higher than that of the male, these values were also higher in the wet season than those in the dry season, and gradually increased from Tra Vinh to $\mathrm{Ca}$ Mau. These values of the Periophthalmus chrysospilos fish were also influenced by the interaction of season $\times$ site and gender $\times$ site. In addition, the morphological variations of this species such as eye diameter $(E D)$, eye distance $(D E)$, body height $(B D)$, head length $(H L)$ and taxonomic ratios such as $H L / T L, B D / T L, E D / H L, D E / H L$ were also affected by gender, seasonal fluctuations, and sites. These morphological variations and ratios were also influenced by interactions season $\times$ site. The results supplied information related to fish identification and the ecological adaptation conditions of this species.
\end{abstract}

Keywords: morphological variations, Tra Vinh, Soc Trang Bac Lieu, Ca Mau, fish identification, ecological adaptation.

\footnotetext{
* Corresponding author.

Địa chi email: dmquang@ctu.edu.vn

https://doi.org/10.25073/2588-1140/vnunst.5245
} 


\title{
Sự biến động một số thông số hình thái của Periophthalmus chrysospilos phân bố ở ven biển Đồng bằng sông Cửu Long
}

\author{
Lê Trung Hiếu ${ }^{1}$, Đinh Minh Quang ${ }^{2, *}$, Hứa Văn Ư ${ }^{3}$, Nguyễn Hữu Đức Tôn ${ }^{2}$ \\ ${ }^{1}$ Trường THPT Trần Đại Nghĩa, Hưng Phú, Cái Răng, Thành Phố Cần Tho, Việt Nam \\ ${ }^{2}$ Đại học Cần Tho, Đường 3/2, Xuân Khánh, Ninh Kiều, Thành phố Cần Tho', Việt Nam \\ ${ }^{3}$ Truoòng THPT chuyên Nguyễn Thị Minh Khai, Số 1 Hồ Nước Ngọt, \\ Phường 6, Thành phố Sóc Trăng, Sóc Trăng, Việt Nam \\ Nhận ngày 30 tháng 5 năm 2021 \\ Chỉnh sửa ngày 24 tháng 7 năm 2021; Chấp nhận đăng ngày 15 tháng 8 năm 2021
}

\begin{abstract}
Tóm tắt: Nghiên cứu này cung cấp dẫn liệu về sự biến đổi hình thái ở cá thòi lòi chấm cam Periophthalmus chrysospilos. Nghiên cứu được khảo sát tại bốn tỉnh ven biển từ Trà Vinh đến Sóc Trăng, Bạc Liêu và Cà Mau kéo dài trong 12 tháng từ 04/2020 đến 03/2021. Kết quả phân tích 1.031 cá thể (508 cái và 523 đực) cho thấy chiều dài tổng $(T L)$ và khối lượng $(W)$ của cá thòi lòi Periophthalmus chrysospilos cái cao hơn cá đực, ở mùa mưa cao hơn so với mùa khô và tăng dần từ Trà Vinh đến Cà Mau. Bên cạnh đó chiều dài tổng và khối lượng cá còn chịu sự ảnh hưởng của tương tác mùa $\times$ địa điểm và giới tính $\times$ địa điểm. Ngoài ra các thông số hình thái của loài này như đường kính mắt $(E D)$, khoảng cách mắt $(D E)$, cao thân $(B D)$, dài đầu $(H L)$ và các tỉ lệ đặc trưng của loài này trong định loại như $H L / T L, B D / T L, E D / H L, D E / H L$ có sự biến động theo giới tính, mùa và điểm nghiên cứu. Các thông số và tỉ lệ này cũng chịu sự ảnh hưởng của các tương tác mùa $\times$ địa điểm. Kết quả nghiên cứu bổ sung thêm thông tin cho định loại cũng như là sự hiểu biết thích nghi sinh thái của loài cá này.
\end{abstract}

Tù khóa: Bạc Liêu, Cà Mau, chỉ số hình thái, định loại, Trà Vinh, Sóc Trăng.

\section{1. Đặt vấn đề}

Theo Strauss và Bond [1], các tỉ lệ có được từ các thông số hình thái như dài tổng $(T L)$, cao thân $(B D)$, dài đầu $(H L)$, đường kính mắt $(E D)$ và khoảng cách hai mắt $(D E), H L / T L, B D / T L$, $E D / H L, D E / H L$ có vai trò trong việc định loại cá, bên cạnh các chỉ số đo và đếm. Các giá trị hình thái này là các thông số cơ bản trong nghiên cứu hình thái và định loại cá [2]. Đây là các thông số cơ bản, dễ dàng nhận biết, xác định trên hầu hết các loài cá. Bên cạnh đó, các thông số này cũng thường xuyên sử dụng trong công tác phân tích sinh học cá $[3,4]$. Ở các môi

\footnotetext{
*Tác giả liên hệ.

Địa chỉ email: dmquang@ctu.edu.vn

https://doi.org/10.25073/2588-1140/vnunst.5245
}

trường khác nhau thì các yếu tố như độ mặn và độ $\mathrm{pH}$ có tác động đến quá trình phát triển của loài cá này cũng như các sinh vật là nguồn thức ăn của chúng, từ đó tác động lên sự sinh trưởng của cá gây nên các thay đổi về hình thái [5-7]. Mối liên hệ của yếu tố môi trường với sự thích nghi sinh thái của cá ở vùng Đồng bằng sông Cửu Long (ĐBSCL) còn chưa được biết đến nhiều, đặc biệt là nhóm cá bùn.

Cá thòi lòi chấm cam Periophthalmus chrysospilos Bleeker 1853 là một trong những loài cá bùn và thường xuất hiện ở bãi bồi các vùng ven biển, đặc biệt các bãi bồi với rừng ngập mặn vùng Ấn Độ đến Indonesia và ĐBSCL [8-10]. Đây là loài cá có môi trường sống khác biệt với đa số các loài cá khác, chúng có thể sống ở nước và môi trường cạn trong một 
khoảng thời gian [11, 12]. Tuy nhiên, vẫn chưa có nghiên cứu mối quan hệ giữa nhân tố môi trường với đặc điểm hình thái của loài cá này. Vì vậy, nghiên cứu này nhằm xác định các thông số hình thái của Ps. chrysospilos có bị thay đổi theo giới tính, mùa và điểm thu mẫu không? Kết quả nghiên cứu bổ sung thêm thông tin về tỉ lệ hình thái có thể sử dụng trong định loại cũng như là sự hiểu biết thích nghi sinh thái của loài này.

\section{Phương pháp nghiên cứu}

\subsection{Thu mẫu và phân tích mẫu}

Mẫu cá Ps. chrysospilos được thu ở bốn điểm ở khu vực ven biển từ Duyên Hải - Trà Vinh (TV) đến Trần Đề - Sóc Trăng (ST), Đông Hải - Bạc Liêu (BL) và Đầm Dơi - Cà Mau (CM) (Hình 1). Mẫu cá (1.031 cá thể) được thu liên tiếp từ tháng $04 / 2020$ đến $03 / 2021$ bằng cách bắt trực tiếp bằng tay ở các bãi bồi ven biển vào lúc chiều tối vì cá di chuyển rất nhanh vào ban ngày. Định kì mẫu được thu mỗi tháng. Mẫu cá sau khi bắt được trữ trong dung dịch formol $10 \%$ và được xử lý tại phòng thí nghiệm. Tại đây, cá được xác định giới tính dựa vào gai sinh dục và vây lưng. Sau đó cá được đo các chỉ tiêu hình thái như: cân khối lượng $(W)$, đo chiều dài tổng $(T L)$, cao thân $(B D)$, dài đầu $(H L)$ đường kính mắt $(E D)$ và khoảng cách hai mắt $(D E)$.

\subsection{Phân tích dĩ liệu}

Sự khác biệt của $T L, W, H L, B D, E D, D E$, $H L / T L, B D / T L, E D / H L$ và $D E / H L$ giữa cá đực và cá cái, mùa khô và mùa mưa được xác định bằng phép thử t-test. Sự thay đổi của các chỉ số này ở các khu vực được xác định bằng phép thử one-way ANOVA. Ngoài ra, phép thử two-way ANOVA để kiểm tra sự tác động của chỉ số giới tính $\times$ mùa, giới tính $\times$ địa điểm nghiên cứu và mùa $\times$ địa điểm nghiên cứu lên sự biến đổi của $T L, H L / T L, B D / T L, E D / H L$ và $D E / H L$. Các giá trị của các thông số được xử lý bởi phần mềm SPSS v21. Các phép thử đều có độ tin cậy $95 \%$.

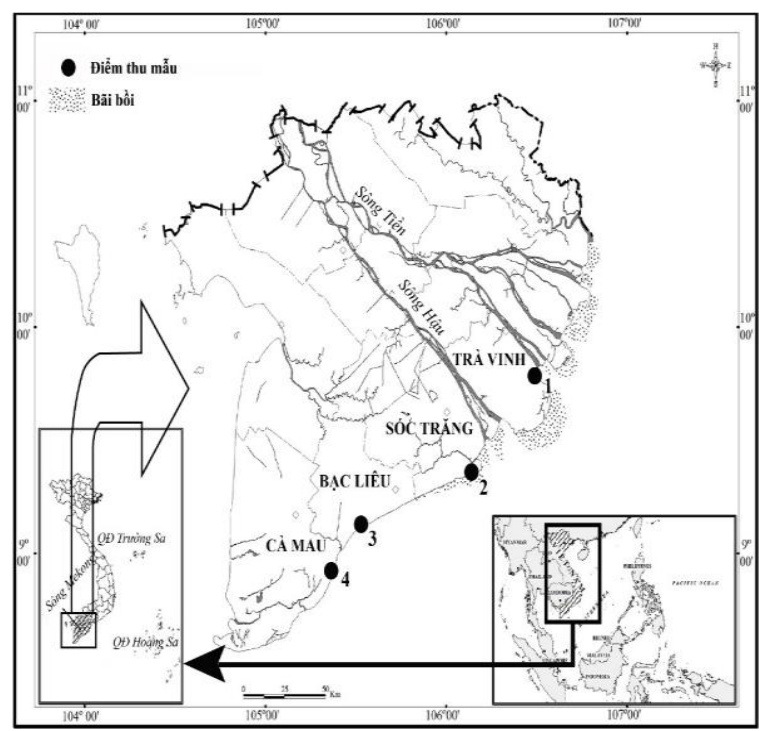

Hình 1. Bản đồ thu mẫu cá Periophthalmus chrysospilos ở ĐBSCL.

( $\bullet$ : Điểm thu mẫu; 1: Duyên Hải - Trà Vinh;

2: Trần Đề - Sóc Trăng; 3: Đông Hải - Bạc Liêu; 4: Đầm Dơi - Cà Mau) [13].

\section{Nghiên cứu và thảo luận}

\subsection{Sự biến động chiều dài và khối lượng}

Kết quả phân tích $T L$ và $W$ của 1.031 cá thể thòi lòi Ps. chrysospilos tại bốn khu vực thu mẫu cho thấy chỉ số này có sự thay đổi theo địa điểm. Chiều dài tổng $(T L)$ của loài này đạt giá trị lớn nhất tại $\mathrm{CM}$ với giá trị trung bình là $5,39 \pm 0,16 \mathrm{SE} \mathrm{cm}$. Tại $\mathrm{ST}$ và $\mathrm{BL}$, cá có chiều dài trung bình tương đương nhau nhưng nhỏ hơn ở $\mathrm{CM}$ với các kết quả là 4,56 $\pm 0,14 \mathrm{SE} \mathrm{cm}$ và $4,58 \pm 0,13 \mathrm{SE} \mathrm{cm}$. Điểm có giá trị thấp nhất là Duyên Hải - Trà Vinh $(\mathrm{TV})$ với giá trị trung bình là $3,31 \pm 0,14 \mathrm{SE} \mathrm{cm}(F=37,68 ; P<0,05$ (Hình 2). Về khối lượng cá cũng có sự biến đổi theo địa điểm one-way ANOVA, $F=35,09$; $P<0,05$ (Hình 3 ). Chỉ số này đạt giá trị lớn nhất tại $\mathrm{CM}$ và giảm dần đến $\mathrm{TV}$ với các kết quả là $8,08 \pm 0,08 \mathrm{SE} ; 7,70 \pm 0,07 \mathrm{SE} ; 7,72 \pm 0,08 \mathrm{SE}$ và $6,87 \pm 0,09 \mathrm{SE}$.

Thay đổi đồng thời của $T L$ và $W$ của cá cho thấy hai chỉ số này có mối sự tăng trưởng như nhau. Bên cạnh đó các chỉ số môi trường đã làm cho hai chỉ số này có sự biến động giữa các địa điểm, đặc biệt là yếu tố độ mặn. Theo nghiên cứu 
của Dinh và các cộng sự [14], độ mặn có sự thay đổi và tăng dần từ $T V$ đến $S T, B L$ và $C M$ với các giá trị lần lượt là 12,$33 ; 14,00 ; 23,50$ và $23,17 \%$.

Độ $\mathrm{pH}$ có giá trị thấp nhất tại $\mathrm{CM}(7,63)$ và cao nhất tại $\operatorname{TV}(7,85)$ và $\mathrm{ST}(7,96)$. Trong nghiên cứu này sự thay đổi các giá trị $T L$ và $W$ có sự tỉ lệ thuận với độ mặn và tỉ lệ nghịch với $\mathrm{pH}$. Qua đó thấy được các chỉ số này có mối quan hệ với các yếu tố môi trường.

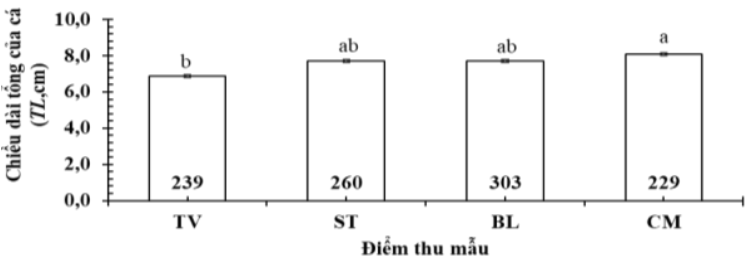

Hình 2. Sự thay đổi chiều dài tổng của cá Periophthalmus chrysospilos ở các điểm nghiên cứu. (TV: Duyên Hải, Trà Vinh; ST: Trần Đề, Sóc Trăng; BL: Đông Hải, Bạc Liêu; CM: Đầm Dơi, Cà Mau; số trong cột: số lượng cá thể ở mỗi điểm; thanh dọc là sai số chuẩn của giá trị trung bình; $\mathrm{a}, \mathrm{b}$ : thể hiện sự khác biệt có ý nghĩa thống kê, $\mathrm{P}<0,05$ ).

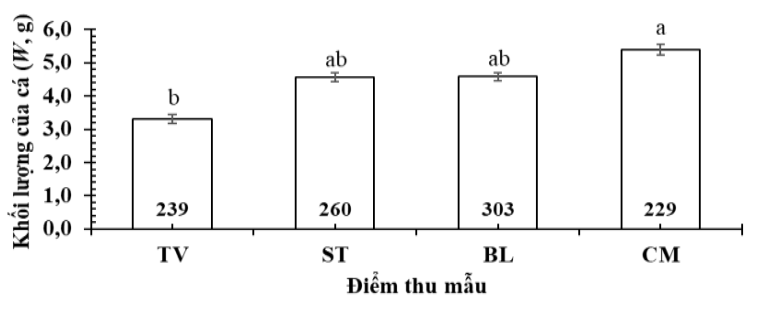

Hình 3. Sự thay đổi khối lượng của cá Periophthalmus chrysospilos ở các điểm nghiên cứu. (TV: Duyên Hải, Trà Vinh; ST: Trần Đề, Sóc Trăng; BL: Đông Hải, Bạc Liêu; CM: Đầm Dơi, Cà Mau; số trong cột: số lượng cá thể ở mỗi điểm; thanh dọc là sai số chuẩn của giá trị trung bình; $\mathrm{a}, \mathrm{b}$ : thể hiện sự khác biệt có ý nghĩa thống kê, $\mathrm{P}<0,05)$.
Ở cá thòi lòi Periophthalmus chrysospilos, $T L$ và $W$ của cá không chỉ thay đổi theo điểm nghiên cứu mà 2 thông số này còn thay đổi theo giới tính và mùa. Giá trị trung bình cho thấy ở cá cái luôn có giá trị trung bình của $T L$ và $W$ cao hơn cá đực (Bảng 1). Cụ thể cá cái có trung bình chiều dài tổng là $7,83 \pm 0,06 \mathrm{SE} \mathrm{cm}$ cao hơn cá đực là 7,38 $\pm 0,06 \mathrm{SE} \mathrm{cm}$ (t-test, $t=5,46$; $P<0,05)$. Cá cái có khối lượng với giá trị trung bình là $4,85 \pm 0,11 \mathrm{SE} \mathrm{g}$, giá trị này ở cá đực là $4,08 \pm 0,10$ SE g $(t=5,40 ; P<0,05)$. Qua đó cho thấy cá cái có sự phát triển mạnh hơn cá đực ở 4 điểm nghiên cứu. Nguyên nhân có thể là do cá cái cần tích lũy năng lượng nhiều hơn cho việc sinh sản. Song song đó, yếu tố mùa cũng có ảnh hưởng mạnh mẽ đến sự tăng trưởng của cá. Bằng chứng là $T L$ và $W$ của cá có giá trị vào mùa mưa lớn hơn mùa khô $\left(t_{T L}=-7,76 ; t_{W}=-5,61\right.$; $P_{T L}<0,05 ; P_{W}<0,05 ;$ Bảng 1$)$. Có thể thấy vào mùa mưa cá có môi trường sống tốt hơn với lượng thức ăn đa dạng hơn và môi trường tương thích hơn nên sự phát triển hình thái của cá mạnh hơn so với mùa khô. Sự thay đổi của những giá trị này theo mùa còn được tìm thấy ở loài Parapocryptes serperaster [15] có cùng họ cá Oxudercidae và phân bố ở ĐBSCL. Tuy nhiên cũng trong họ cá này nhưng Periophthalmodon [16], Trypauchen vagina [17] và Boleophthalmus boddarti [18] không có sự thay đổi $T L$ và $W$ theo mùa. Các loài cá thuộc họ Gobiidae và Butidae như Glossogobius sparsipapillus [19], Glossogobius giuris [20], Glossogobius aureus [21] và Butis koilomatodon [22] cùng khu vực phân bố cũng cho kết quả tương tự.

Bảng 1. Sự thay đổi chiều dài và khối lượng cá thòi lòi Periophthalmus chrysospilos theo giới tính và mùa

\begin{tabular}{|l|c|c|c|}
\hline Thông số hình thái & Nhóm & Số lượng & Trung bình \pm SE \\
\hline \multirow{2}{*}{ Chiều dài tổng } & Cá cái & 508 & $7,83 \pm 0,06^{\mathrm{a}}$ \\
\cline { 2 - 4 } & Cá đực & 523 & $7,38 \pm 0,06^{\mathrm{b}}$ \\
\hline \multirow{2}{*}{ Khối lượng } & Cá cái & 508 & $4,85 \pm 0,11^{\mathrm{a}}$ \\
\cline { 2 - 4 } & Cá đực & 523 & $4,08 \pm 0,10^{\mathrm{b}}$ \\
\hline \multirow{2}{*}{ Chiều dài tổng } & Mùa khô & 408 & $7,21 \pm 0,08^{\mathrm{b}}$ \\
\cline { 2 - 4 } & Mùa mưa & 623 & $7,85 \pm 0,05^{\mathrm{a}}$ \\
\hline \multirow{2}{*}{ Khối lượng } & Mùa khô & 408 & $3,97 \pm 0,13^{\mathrm{b}}$ \\
\cline { 2 - 4 } & Mùa mưa & 623 & $4,79 \pm 0,08^{\mathrm{a}}$ \\
\hline
\end{tabular}




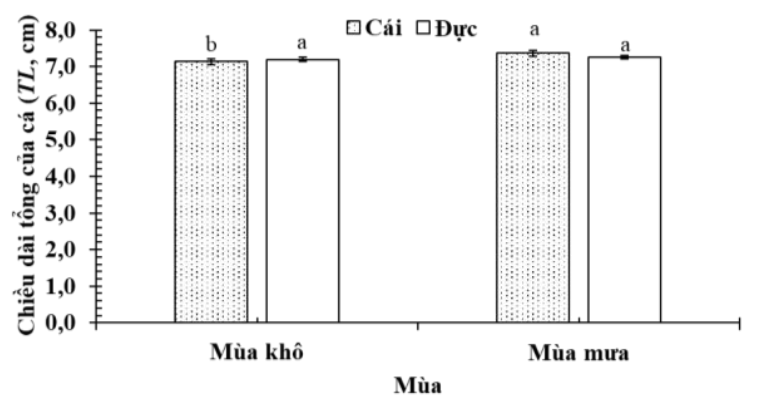

Hình 4. Sự thay đổi chiều dài tổng của cá Periophthalmus chrysospilos theo giới tính và mùa. (thanh dọc là sai số chuẩn của giá trị trung bình; $a, b$ : thể hiện sự khác biệt có ý nghĩa thống kê, $\mathrm{P}<0,05$ )

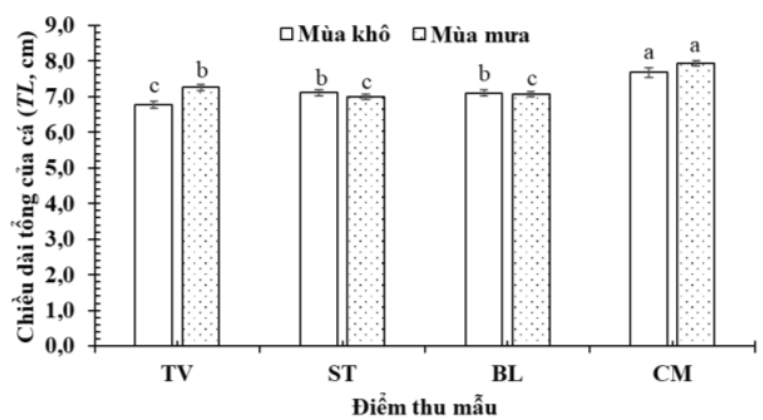

Hình 5. Sự thay đổi chiều dài tổng của cá Periophthalmus chrysospilos theo mùa và địa điểm (TV: Duyên Hải, Trà Vinh; ST: Trần Đề, Sóc Trăng; BL: Đông Hải, Bạc Liêu; CM: Đầm Dơi, Cà Mau; thanh dọc là sai số chuẩn của giá trị trung bình; $a, b, c$ : thể hiện sự khác biệt có ý nghĩa thống kê, $\mathrm{P}<0,05)$.

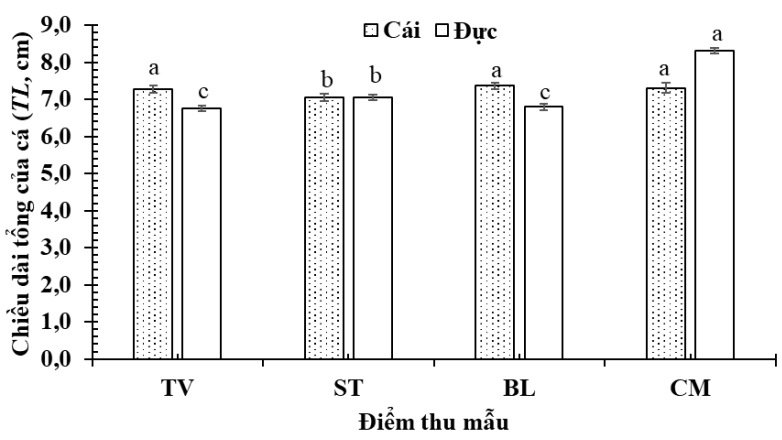

Hình 6. Sự thay đổi chiều dài tổng của cá Periophthalmus chrysospilos theo giới tính và địa điểm. (TV: Duyên Hải, Trà Vinh; ST: Trần Đề, Sóc Trăng; BL: Đông Hải, Bạc Liêu; CM: Đầm Dơi, Cà Mau; thanh dọc là sai số chuẩn của giá trị trung bình; $a, b, c$ : thể hiện sự khác biệt có ý nghĩa thống kê, $\mathrm{P}<0,05$ ).

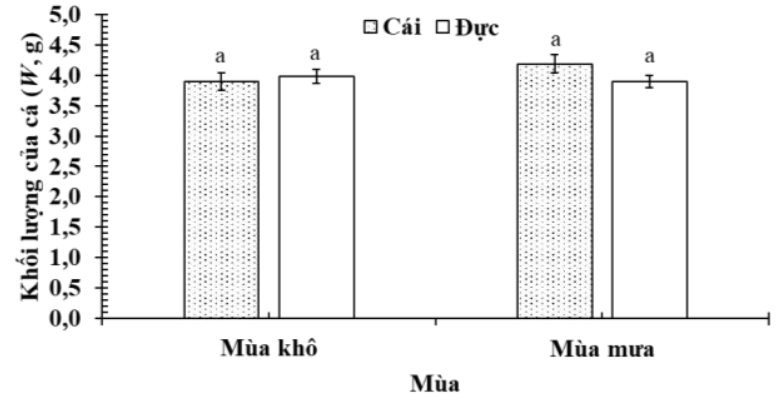

Hình 7. Sự thay đổi khối lượng của cá Periophthalmus chrysospilos ở theo giới tính và mùa (thanh dọc là sai số chuẩn của giá trị trung bình; $\mathrm{a}, \mathrm{b}$ : thể hiện sự khác biệt có ý nghĩa thống kê, $\mathrm{P}<0,05)$.

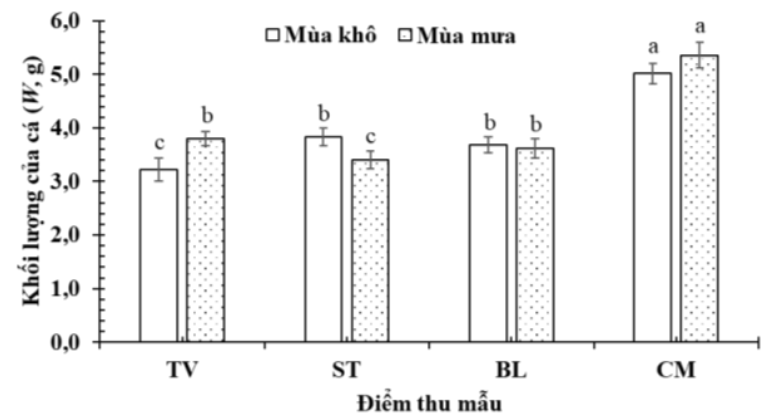

Hình 8. Sự thay đổi khối lượng cá Periophthalmus chrysospilos theo mùa và địa điểm

(TV: Duyên Hải, Trà Vinh; ST: Trần Đề, Sóc Trăng;

BL: Đông Hải, Bạc Liêu; CM: Đầm Dơi, Cà Mau;

thanh dọc là sai số chuẩn của giá trị trung bình; $a, b, c$ : thể hiện sự khác biệt có ý nghĩa thống kê, $\mathrm{P}<0,05)$.

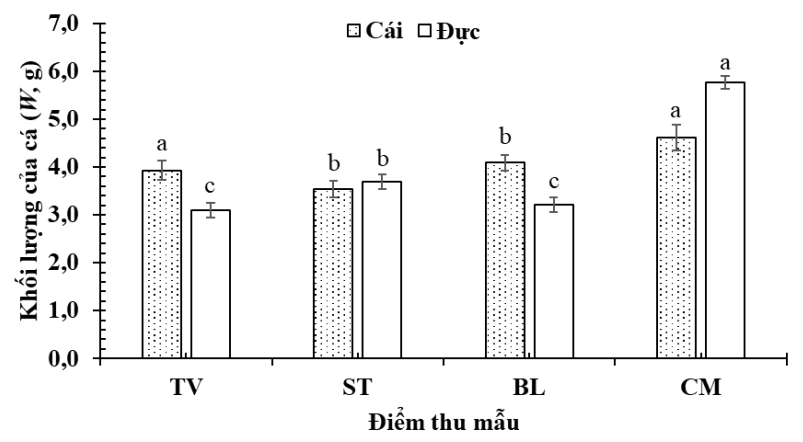

Hình 9. Sự thay đổi khối lượng cá Periophthalmus chrysospilos theo giới tính và địa điểm.

(TV: Duyên Hải, Trà Vinh; ST: Trần Đề, Sóc Trăng; BL: Đông Hải, Bạc Liêu; CM: Đầm Dơi, Cà Mau; thanh dọc là sai số chuẩn của giá trị trung bình; $a, b, c$ : thể hiện sự khác biệt có ý nghĩa thống kê, $\mathrm{P}<0,05)$. 
3.2. Sự biến động đường kính mắt, khoảng cách mắt, chiều cao thân và chiều dài đầu và các tỉ lệ hình thái

Ngoài sự biến động về $T L$ và $W$ theo giới tính, mùa và địa điểm thì cá thòi lòi Periophthalmus chrysospilos còn có sự thay đổi các thông số hình thái khác như: khoảng cách hai mắt $(D E)$, đường kính mắt $(E D)$, cao thân $(B D)$, dài đầu $(H L)$. Các thông số này tạo nên các tî lệ chiều dài đầu/chiều dài tổng $(H L / T L)$, chiều cao thân/chiều dài tổng $(B D / T L)$, đường kính mắt/ chiều dài đầu $(E D / H L)$, khoảng cách mắt/chiều dài đầu $(D E / H L)$. Các tỉ lệ này ở mỗi loài cá có sự khác biệt và đặc trưng cho loài. Ở loài cá này cá thông số hình thái của cá và các tỉ lệ đặc trưng đều có sự chênh lệch giữa cá cái và cá đực (ngoại trừ $D E$ và $B D / T L)(P>0,05$ cho mọi trường hợp, Bảng 2). So với loài G.aureus, các tỉ lệ $H L / T L, B D / T L$ và $E D / H L$ ở loài cá này đều có giá trị nhỏ hơn. Ngược lại , $D E / H L$ ở loài này lớn hơn khá nhiều so với $G$. aureus [21]. Trong khi đó ở loài G. giuris cùng giống Glossogobius với $G$. aureus, có $H L / T L$ và $D E / H L$ có giá trị lần lượt là 0,18 và 0,23 . Cả hai giá trị này đều thấp hơn so với $P$. chrysospilos. Ở hai tỉ lệ còn lại thì loài G. giuris có giá trị cao hơn loài này [20]. Qua đó cho thấy mổi loài đều có các giá trị tỉ lệ hình thái khác nhau. Do các loài khác trong họ Periophthalmus tại ĐBSCL cũng như trên thế giới vẫn chưa được nghiên cứu nên vẫn chưa thể xác định các giá trị hình thái giữa các loài này có khác nhau hay không. Tuy nhiên, kết quả này có thể dùng để so sánh với các loài trong giống này ở các nghiên cứu tiếp theo.

Bảng 2. Sự biến động các thông số hình thái của cá thòi lòi Periophthalmus chrysospilos theo giới tính

\begin{tabular}{|c|c|c|c|c|c|c|}
\hline $\begin{array}{l}\text { Thông số } \\
\text { hình thái }\end{array}$ & Giới tính & Số lượng & Trung bình & Sai số chuẩn & $t$ & $P$ \\
\hline \multirow{2}{*}{$E D$} & Cá cái & 508 & 0,373 & 0,003 & \multirow{2}{*}{4,88} & \multirow{2}{*}{0,00} \\
\hline & Cá đực & 523 & 0,353 & 0,003 & & \\
\hline \multirow{2}{*}{$D E$} & Cá cái & 508 & 0,187 & 0,003 & \multirow{2}{*}{2,76} & \multirow{2}{*}{0,06} \\
\hline & Cá đực & 523 & 0,176 & 0,003 & & \\
\hline \multirow{2}{*}{$B D$} & Cá cái & 508 & 0,964 & 0,012 & \multirow{2}{*}{5,02} & \multirow{2}{*}{0,00} \\
\hline & Cá đực & 523 & 0,880 & 0,012 & & \\
\hline \multirow{2}{*}{$H L$} & Cá cái & 508 & 1,695 & 0,016 & \multirow{2}{*}{2,99} & \multirow{2}{*}{0,03} \\
\hline & Cá đực & 523 & 1,631 & 0,015 & & \\
\hline \multirow{2}{*}{$H L / T L$} & Cá cái & 508 & 0,229 & 0,003 & \multirow{2}{*}{1,97} & \multirow{2}{*}{0,05} \\
\hline & Cá đực & 523 & 0,221 & 0,002 & & \\
\hline \multirow{2}{*}{$B D / T L$} & Cá cái & 508 & 0,115 & 0,002 & \multirow{2}{*}{1,53} & \multirow{2}{*}{0,12} \\
\hline & Cá đực & 523 & 0,111 & 0,002 & & \\
\hline \multirow{2}{*}{$E D / H L$} & Cá cái & 508 & 0,122 & 0,001 & \multirow{2}{*}{2,40} & \multirow{2}{*}{0,02} \\
\hline & Cá đực & 523 & 0,118 & 0,001 & & \\
\hline \multirow{2}{*}{$D E / H L$} & Cá cái & 508 & 0,272 & 0,001 & \multirow{2}{*}{$-3,26$} & \multirow{2}{*}{0,02} \\
\hline & Cá đực & 523 & 0,278 & 0,001 & & \\
\hline
\end{tabular}

Giống với sự thay đổi theo giới tính, hầu hết các thông số hình thái ở cá thòi lòi Periophthalmus chrysospilos có sự biến động theo mùa, ngoại trừ thông số về khoảng cách mắt ( $P<0,05$ cho mọi trường hợp, Bảng 3$)$. Cụ thể, vào mùa mưa thì các thông số của cá đều cao hơn so với mùa khô. Qua đó cho thấy vào mùa mưa thuận lợi hơn cho sự phát triển của 
loài cá này. Từ sự thay đổi của các thông số hình thái dẫn đến các tỉ lệ đặc trưng ở loài cá này cũng có sự thay đổi theo mùa $(P<0,05$ cho mọi trường hợp, Bảng 3$)$. Các giá trị $E D, B D$ và
$H L$ đều tăng vào mùa mưa nhưng và gây ảnh hưởng đến giá trị của các tỉ lệ $B D / T L, H L / T L$ $E D / H L$ và $D E / H L$. Qua đó thấy được sự thay đổi của hai giá trị hình thái ở mỗi cặp tỉ lệ.

Bảng 3. Sự biến động các thông số hình thái của cá thòi lòi Periophthalmus chrysospilos theo mùa

\begin{tabular}{|c|c|c|c|c|c|c|}
\hline Thông số hình thái & Mùa & Số lượng & Trung bình & Sai số chuẩn & $t$ & $P$ \\
\hline \multirow{2}{*}{$E D$} & Mùa khô & 408 & 0,355 & 0,003 & \multirow{2}{*}{$-3,16$} & \multirow{2}{*}{0,02} \\
\hline & Mùa mưa & 623 & 0,368 & 0,003 & & \\
\hline \multirow{2}{*}{$D E$} & Mùa khô & 408 & 0,185 & 0,004 & \multirow{2}{*}{1,33} & \multirow{2}{*}{0,18} \\
\hline & Mùa mưa & 623 & 0,179 & 0,002 & & \\
\hline \multirow{2}{*}{$B D$} & Mùa khô & 408 & 0,848 & 0,014 & \multirow{2}{*}{$-7,16$} & \multirow{2}{*}{0,00} \\
\hline & Mùa mưa & 623 & 0,969 & 0,010 & & \\
\hline \multirow{2}{*}{$H L$} & Mùa khô & 408 & 1,575 & 0,019 & \multirow{2}{*}{$-6,65$} & \multirow{2}{*}{0,00} \\
\hline & Mùa mưa & 623 & 1,719 & 0,012 & & \\
\hline \multirow{2}{*}{$H L / T L$} & Mùa khô & 408 & 0,233 & 0,003 & \multirow{2}{*}{3,46} & \multirow{2}{*}{0,01} \\
\hline & Mùa mưa & 623 & 0,220 & 0,002 & & \\
\hline \multirow{2}{*}{$B D / T L$} & Mùa khô & 408 & 0,122 & 0,003 & \multirow{2}{*}{5,05} & \multirow{2}{*}{0,00} \\
\hline & Mùa mưa & 623 & 0,107 & 0,001 & & \\
\hline \multirow{2}{*}{$E D / H L$} & Mùa khô & 408 & 0,116 & 0,001 & \multirow{2}{*}{$-4,57$} & \multirow{2}{*}{0,00} \\
\hline & Mùa mưa & 623 & 0,123 & 0,001 & & \\
\hline \multirow{2}{*}{$D E / H L$} & Mùa khô & 408 & 0,277 & 0,001 & \multirow{2}{*}{2,22} & \multirow{2}{*}{0,00} \\
\hline & Mùa mưa & 623 & 0,273 & 0,001 & & \\
\hline
\end{tabular}

Tại mỗi khu vực đều có đặc trưng về các yếu tố môi trường (độ mặn và độ $\mathrm{pH}$ ) nên sự biến động các thông số hình thái và các tỉ lệ đặc trưng có sự khác nhau ở mỗi địa điểm $(P<0,05$ cho mọi trường hợp, Bảng 4). Hầu hết các giá trị cao về các thông số hình thái đều thuộc khu vực Đầm Dơi - Cà Mau và thấp nhất là khu vực Duyên Hải - Trà Vinh (Ngoại trừ $D E$ ). Từ đó cho thấy các yếu tố môi trường tại các khu vực này (độ mặn cao và $\mathrm{pH}$ thấp) tương thích với sự sinh trưởng của cá. Bên cạnh đó, trong quá trình quan sát khi thu mẫu thì đây là các khu vực ít chịu sự ảnh hưởng từ con người nên cá có môi trường phát triển ổn định. Ở mỗi môi trường sống đặc trưng cho các giá trị hình thái khác nhau ở loài cá này tương tự với sự biến đổi các thông số hình thái ở loài Glossogobius sparsipapillus [19], Glossogobius giuris [20], Glossogobius aureus [21], Butis koilomatodon [22], Parapocryptes serperaster [15] và Periophthalmodon septemradiatus [23]. Qua đó cho thấy, tuy ở mỗi loài cá các giá trị hình thái riêng lẻ có thể thay đổi theo từng môi trường sống của cá. Nhưng khi xét trên các tỉ lệ hình thái thì giữa các điểm nghiên cứu của các loài thì sự thay đổi này không lớn. Điều này chứng tỏ các tỉ lệ hình thái có sự đặc trưng cho từng loài, trong đó có Periophthalmus chrysospilos. Giá trị trung bình của các tỉ lệ này đều nhỏ hơn so với tỉ lệ của loài Periophthalmodon septemradiatus [23] và Parapocryptes serperaster [15].

Tương tự như $T L$ và $W$, các thông số hình thái của cá thòi Periophthalmus chrysospilos không chịu sự tác động cùng lúc của mùa $\times$ giới tính, mùa $\times$ địa điểm, ngoại trừ $D E$ và $B D$ có biến đổi bởi sự ảnh hưởng của mùa $\times$ địa điểm. Các thông số hình thái của loài này còn chịu sự tác động của giới tính $\times$ địa điểm. Bên cạnh các thông số hình thái, các tî̉ lệ đặc trưng của cá thòi lòi Periophthalmus chrysospilos không chịu sự tác động của mùa $\times$ giới tính, giới tính $\times$ địa điểm và mùa $\times$ địa điểm. Ngoại trừ tỉ lệ $B D / T L, E D / H L$ và $D E / H L$ thay đồi theo mùa $\times$ địa điểm (Bảng 5). 
Bảng 4. Sự biến động các thông số hình thái của cá thòi lòi Periophthalmus chrysospilos theo địa điểm

\begin{tabular}{|c|c|c|c|c|c|c|}
\hline $\begin{array}{l}\text { Thông số } \\
\text { hình thái }\end{array}$ & Điểm & Số lượng & $\begin{array}{l}\text { Trung } \\
\text { bình }\end{array}$ & $\begin{array}{l}\text { Sai số } \\
\text { chuẩn }\end{array}$ & $F$ & $P$ \\
\hline \multirow{4}{*}{$E D$} & Duyên Hải, Trà Vinh & 239 & 0,338 & 0,003 & \multirow{4}{*}{17,47} & \multirow{4}{*}{0,00} \\
\hline & Trần Đề, Sóc Trăng & 260 & 0,369 & 0,004 & & \\
\hline & Đông Hải, Bạc Liêu & 303 & 0,362 & 0,004 & & \\
\hline & Đầm Dơi, Cà Mau & 229 & 0,381 & 0,004 & & \\
\hline \multirow{4}{*}{$D E$} & Duyên Hải, Trà Vinh & 239 & 0,192 & 0,005 & \multirow{4}{*}{13,93} & \multirow{4}{*}{0,00} \\
\hline & Trần Đề, Sóc Trăng & 260 & 0,186 & 0,003 & & \\
\hline & Đông Hải, Bạc Liêu & 303 & 0,161 & 0,003 & & \\
\hline & Đầm Dơi, Cà Mau & 229 & 0,191 & 0,003 & & \\
\hline \multirow{4}{*}{$B D$} & Duyên Hải, Trà Vinh & 239 & 0,796 & 0,013 & \multirow{4}{*}{33,32} & \multirow{4}{*}{0,00} \\
\hline & Trần Đề, Sóc Trăng & 260 & 0,974 & 0,016 & & \\
\hline & Đông Hải, Bạc Liêu & 303 & 0,900 & 0,015 & & \\
\hline & Đầm Dơi, Cà Mau & 229 & 1,018 & 0,018 & & \\
\hline \multirow{4}{*}{$H L$} & Duyên Hải, Trà Vinh & 239 & 1,475 & 0,021 & \multirow{4}{*}{35,46} & \multirow{4}{*}{0,00} \\
\hline & Trần Đề, Sóc Trăng & 260 & 1,714 & 0,021 & & \\
\hline & Đông Hải, Bạc Liêu & 303 & 1,685 & 0,019 & & \\
\hline & Đầm Dơi, Cà Mau & 229 & 1,766 & 0,019 & & \\
\hline \multirow{4}{*}{$H L / T L$} & Duyên Hải, Trà Vinh & 239 & 0,236 & 0,003 & \multirow{4}{*}{4,21} & \multirow{4}{*}{0,01} \\
\hline & Trần Đề, Sóc Trăng & 260 & 0,222 & 0,003 & & \\
\hline & Đông Hải, Bạc Liêu & 303 & 0,223 & 0,004 & & \\
\hline & Đầm Dơi, Cà Mau & 229 & 0,217 & 0,002 & & \\
\hline \multirow{4}{*}{$B D / T L$} & Duyên Hải, Trà Vinh & 239 & 0,136 & 0,004 & \multirow{4}{*}{31,69} & \multirow{4}{*}{0,00} \\
\hline & Trần Đề, Sóc Trăng & 260 & 0,113 & 0,002 & & \\
\hline & Đông Hải, Bạc Liêu & 303 & 0,096 & 0,001 & & \\
\hline & Đầm Dơi, Cà Mau & 229 & 0,109 & 0,002 & & \\
\hline \multirow{4}{*}{$E D / H L$} & Duyên Hải, Trà Vinh & 239 & 0,115 & 0,001 & \multirow{4}{*}{13,59} & \multirow{4}{*}{0,00} \\
\hline & Trần Đề, Sóc Trăng & 260 & 0,125 & 0,001 & & \\
\hline & Đông Hải, Bạc Liêu & 303 & 0,115 & 0,001 & & \\
\hline & Đầm Dơi, Cà Mau & 229 & 0,125 & 0,001 & & \\
\hline \multirow{4}{*}{$D E / H L$} & Duyên Hải, Trà Vinh & 239 & 0,271 & 0,002 & \multirow{4}{*}{3,88} & \multirow{4}{*}{0,01} \\
\hline & Trần Đề, Sóc Trăng & 260 & 0,279 & 0,002 & & \\
\hline & Đông Hải, Bạc Liêu & 303 & 0,274 & 0,002 & & \\
\hline & Đầm Dơi, Cà Mau & 229 & 0,272 & 0,001 & & \\
\hline
\end{tabular}

Bảng 5. Sự biến động các thông số hình thái của cá thòi lòi Periophthalmus chrysospilos ở hai nhân tố

\begin{tabular}{|l|l|r|r|r|r|r|r|r|r|}
\hline \multicolumn{2}{|l|}{ Tương tác } & $\boldsymbol{E D}$ & $\boldsymbol{D E}$ & $\boldsymbol{B D}$ & $\boldsymbol{H L}$ & $\boldsymbol{H L} / \boldsymbol{T L}$ & $\boldsymbol{B D} / \boldsymbol{T L}$ & $\boldsymbol{E D} / \boldsymbol{H L}$ & $\boldsymbol{D E} / \boldsymbol{H L}$ \\
\hline \multirow{2}{*}{ Giới tính $\times$ mùa } & $F$ & 0,00 & 0,34 & 3,77 & 0,03 & 0,60 & 0,77 & 2,98 & 0,18 \\
\cline { 2 - 10 } & $P$ & 1,00 & 0,56 & 0,05 & 0,86 & 0,44 & 0,38 & 0,09 & 0,67 \\
\hline \multirow{2}{*}{ Giới tính $\times$ địa điểm } & $F$ & 13,29 & 2,26 & 6,01 & 13,63 & 0,11 & 0,13 & 2,21 & 1,41 \\
\cline { 2 - 10 } & $P$ & 0,00 & 0,08 & 0,00 & 0,00 & 0,95 & 0,95 & 0,09 & 0,24 \\
\hline \multirow{2}{*}{ Mùa $\times$ địa điểm } & $F$ & 2,12 & 14,09 & 2,67 & 1,15 & 0,47 & 16,28 & 5,72 & 2,84 \\
\cline { 2 - 10 } & $P$ & 0,10 & 0,00 & 0,05 & 0,33 & 0,70 & 0,00 & 0,00 & 0,04 \\
\hline
\end{tabular}




\section{Kết luận}

Periophthalmus chrysospilos có chiều dài và khối lượng thay đổi theo giới tính, mùa và địa điểm, và sự tác động đồng thời của giới tính $\times$ địa điểm, mùa $\times$ địa điểm. Các thông số hình thái và tỉ lệ đặc trưng của chúng thay đổi theo giới tính, mùa, địa điểm và sự tác động đồng thời của mùa $\times$ địa điểm. Kết quả nghiên cứu bổ sung thêm thông tin về các tỉ lệ hình thái cho định loại cũng như là sự hiểu biết thích nghi sinh thái của loài này.

\section{Lời cảm ơn}

Nghiên cứu này được tài bởi Quỹ Khoa học và Công nghệ Quốc Gia (Nafosted) với mã số đề tài: 106.05-2019.306. Chúng tôi xin cảm ơn Trần Chí Cảnh, Nguyễn Thị Thuý Hiền và Võ Thị Thảo Lam đã hỗ trợ trong quá trình phân tích mẫu.

\section{Tài liệu tham khảo}

[1] R. E. Strauss, C. E. Bond, Taxonomic Methods: Morphology, in: C. B. Schreck, P. B. Moyle (eds), Methods for Fish Biology, American Fisheries Society, Maryland, 1990, pp. 109-140.

[2] C. J. Grant, A. V. Spain, Variation in the Body Shape of Three Species of Australian Mullets (Pisces: Mugilidae) During the Course of Development, Marine and Freshwater Research, Vol. 26, 1977, pp. 723-738.

[3] V. H. Nguyen, S. V. Ngo, Fresh Warter Fish of Vietnam, Agriculture, III, Hanoi, 2001 (in Vietnamese).

[4] Y. D. Mai, T. V. Nguyen, T. V. Nguyen, Y. H. Le, L. B. Hua, Identification of Freshwater Fishes of South Vietnam, Science and Technology Publishing House, Hanoi, 1992 (in Vietnamese).

[5] S. X. Cadrin, V. M. Silva, Morphometric Variation of Yellowtail Flounder, ICES Journal of Marine Science, Vol. 62, 2005, pp. 683-694.

[6] M. Chaklader, M. Siddik, A. Nahar, Taxonomic Diversity of Paradise Threadfin Polynemus Paradiseus (Linnaeus, 1758) Inhabiting Southern Coastal Rivers in Bangladesh, Sains Malaysiana, Vol. 44, 2015, pp. 1241-1248.

[7] M. Siddik, M. Chaklader, M. Hanif, M. Islam, M. Sharker, M. Rahman, Stock Identification of
Critically Endangered Olive Barb, Puntius Sarana (Hamilton, 1822) with Emphasis on Management Implications, Journal of Aquaculture Research and Development, Vol. 7, 2016, pp. 1-6, https://doi.org/10.4172/2155-9546.1000411.

[8] E. O. Murdy, A Taxonomic Revision and Cladistic Analysis of the Oxudercine Gobies (Gobiidae, Oxudercinae), Australian Museum Journal, Vol. 11, 1989, pp. 93, https://doi.org/10.3853/j.0812-7387.11.1989.93.

[9] R. Froese, D. Pauly, FishBase, World Wide Web electronic publication, www.fishbase.org/, 2021, (accessed on: June 10 ${ }^{\text {th }}, 2021$ ).

[10] D. D. Tran, V. T. Nguyen, H. T. M. To, T. T. Nguyen, Q. M. Dinh, Species Composition and Biodiversity Index of Gobiid Assemblage in Estuarine Areas of the Mekong Delta, Vietnam, Egyptian Journal of Aquatic Biology and Fisheries, Vol. 24, 2020, pp. 931-941, https://doi.org/10.21608/ejabf.2020.131385.

[11] G. Polgar, G. Crosa, Multivariate Characterisation of the Habitats of Seven Species of Malayan Mudskippers (Gobiidae: Oxudercinae), Marine Biology, Vol. 156, 2009, pp. 1475-1486, https://doi.org/10.1007/s00227-009-1187-0.

[12] W. P. Low, D. J. W. Lane, Y. K. Ip, A Comparative Study of Terrestrial Adaptations of the Gills in Three Mudskippers: Periophthalmus Chrysospilos, Boleophthalmus Boddarti, and Periophthalmodon Schlosseri, Biological Bulletin, Vol. 175, 1988, pp. 434-438, https://doi.org/10.2307/1541736.

[13] Q. M. Dinh, Aspects of Reproductive Biology of the Red Goby Trypauchen Vagina (Gobiidae) from the Mekong Delta, Journal of Applied Ichthyology, Vol. 34, 2018, pp. 103-110, https://doi.org/10.1111/jai.13521.

[14] Q. M. Dinh, T. T. H. Lam, T. H. D. Nguyen, T. M. Nguyen, T. T. K. Nguyen, N. T. Nguyen, First Reference on Reproductive Biology of Butis Koilomatodon in Mekong Delta, Vietnam, BMC Zoology, Vol. 6, 2021, pp. 1-14, https://doi.org/10.1186/s40850-021-00072-y.

[15] Q. M. Dinh, J. G. Qin, S. Dittmann, D. D. Tran, Morphometric Variation of Parapocryptes Serperaster (Gobiidae) in Dry and Wet Seasons in the Mekong Delta, Vietnam, Ichthyological Research, Vol. 63, 2016, pp. 267-274, https://doi.org/10.1007/s10228-015-0497-0.

[16] Q. M. Dinh, Growth and Body Condition Variation of the Giant Mudskipper Periophthalmodon Schlosseri in Dry and Wet Seasons, Journal of Biology, Vol. 38, 2016, pp. 352-358, https://doi.org/10.15625/ 0866-7160/v38n3.7425. 
[17] Q. M. Dinh, Growth Pattern and Body Condition of Trypauchen Vagina in the Mekong Delta, Vietnam, The Journal of Animal and Plant Sciences, Vol. 26, 2016, pp. 523-531.

[18] Q. M. Dinh, Morphometric, Growth and Condition Factor Variations of Boleophthalmus Boddarti in the Mekong Delta, Vietnam, Iranian Journal of Fisheries Sciences, Vol. 16, 2017, pp. 822-831.

[19] T. H. D. Nguyen, H. T. T. Nguyen, T. C. Tran, Y. T. N. Nguyen, Q. M. Dinh, Morphometric and Meristic Variations of Glossogobius Sparsipapillus along the Coastline in the Mekong Delta, Vietnam, International Journal of Zoology and Animal Biology, Vol. 3, 2020, pp. 1-9, https://doi.org/10.3853/j.08127387.11.1989.93.10.23 880/izab-16000211.

[20] T. H. D. Nguyen, Q. M. Dinh, Morphometric and Meristic Variations in Glossogobius Giuris Distributed in Different Locations in the Mekong
Delta, TNU Journal of Science and Technology, Vol. 226, 2021, pp. 31-38,

https://doi.org/10.3853/j.08127387.11.1989.93.10.34

238/tnu-jst.4274 (in Vietnamese).

[21] G. H. Phan, Q. M. Dinh, N. T. Truong, T. H. D. Nguyen, Variation in Morphometric Characteristics of Glossogobius Aureus Distributed from Can Tho to $\mathrm{Ca}$ Mau, Vietnam Agricultural Science Journal, Vol. 19, 2021, pp. 863-874 (in Vietnamese).

[22] T. T. H. Lam, Q. M. Dinh, Morphometric and Meristic Variability in Butis Koilomatodon in Estuarine and Coastal Areas of the Mekong Delta, Vietnam Agricultural Science Journal, Vol. 3, 2020, pp. 806-816.

[23] Q. M. Dinh, T. L. Tran, T. Y. N. Nguyen, The Flexibility of Morphometric and Meristic Measurements of Periophthalmodon Septemradiatus (Hamilton, 1822) in Hau River, Journal of Science and Technology, Vol. 187, 2018, pp. 81-90. 